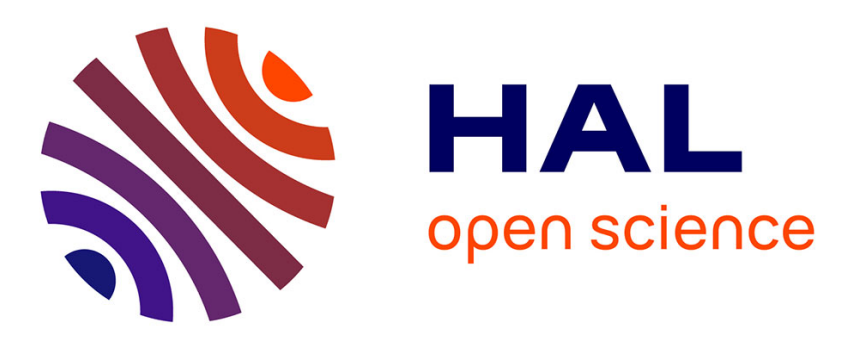

\title{
Impact of bariatric surgery on type 2 diabetes: contribution of inflammation and gut microbiome?
}

Jean Debédat, Chloé Amouyal, Karine Clement, Judith Aron-Wisnewsky

\section{To cite this version:}

Jean Debédat, Chloé Amouyal, Karine Clement, Judith Aron-Wisnewsky. Impact of bariatric surgery on type 2 diabetes: contribution of inflammation and gut microbiome?. Seminars in Immunopathology, 2019, 41 (4), pp.461-475. 10.1007/s00281-019-00738-3 . hal-02291846

\section{HAL Id: hal-02291846 \\ https: / hal.sorbonne-universite.fr/hal-02291846}

Submitted on 19 Sep 2019

HAL is a multi-disciplinary open access archive for the deposit and dissemination of scientific research documents, whether they are published or not. The documents may come from teaching and research institutions in France or abroad, or from public or private research centers.
L'archive ouverte pluridisciplinaire $\mathbf{H A L}$, est destinée au dépôt et à la diffusion de documents scientifiques de niveau recherche, publiés ou non, émanant des établissements d'enseignement et de recherche français ou étrangers, des laboratoires publics ou privés. 


\section{Title page}

Full title: Impact of bariatric surgery on type 2 diabetes: contribution of inflammation and gut microbiome?

\section{Authors}

Jean Debédat*, PharmD ${ }^{1,2}$, Chloé Amouyal*, MD ${ }^{1,2}$, Judith Aron-Wisnewsky ${ }^{\$}$, MD$\mathrm{PhD}^{1,2,3}$, Karine Clément ${ }^{\S} \mathrm{MD}-\mathrm{PhD}^{1,2,3+}$,

- Authors with * contributed equally in the manuscript

- Authors with \$ contributed equally in the manuscript

\section{ORCID:}

- Jean Debédat: 0000-0002-8377-5965

- Chloé Amouyal: 0000-0002-7891-2101

- Judith Aron-Wisnewsky: 0000-0002-3104-9769

- Karine Clément: 0000-0003-4619-6785

\section{Corresponding Authors:}

Karine Clément, MD-PhD ${ }^{1,2,9}$ (karine.clement@inserm.fr)

Address: 47-83 Bd de l'hôpital, 75013 Paris

Phone: + 33142177031

Judith Aron-Wisnewsky, MD-PhD ${ }^{1,2,9}$ (judith.aron-wisnewsky@psl.aphp.fr)

Address: 47-83 Bd de l'hôpital, 75013 Paris

Phone: + 33142177541

\section{Affiliations :}

1. Sorbonne Université, INSERM, Nutrition and Obesities ; Systemic approaches (NutriOmics), Paris, France

2. Assistance Publique Hôpitaux de Paris, Nutrition Departement, Pitié-Salpêtrière Hospital, Sorbonne université Paris, France 


\section{Authors contributions}

JD and CA contributed to the research, discussion of content, writing of this manuscript, J.A.W contributed to the research, discussion of content, writing and editing of this manuscript, and K.C. contributed to the discussion of content, writing and reviewing/editing the manuscript before submission. All authors reviewed the manuscript.

\section{Financial support}

Funding to support NutriOmics research Unit activity on this reviewed topic were obtained from European Union's Seventh Framework Program (FP7) for research, technological development and demonstration under grant agreement HEALTH-F4-2012305312 (Metacardis) and from the French national program "Investissement d'Avenir" FORCE, the Metagenopolis grant ANR-11-DPBS-0001 and the ANR-10-IAHU-05, (Institute of Cardiometabolism and Nutrition), from the National Agency of Research ANR-OB MAIT, CAPTOR and from INSERM (to CA as program “Accueil Jeune Chercheur”). JAW received grant from Institut Benjamin Delessert, and Société Francophone du Diabète (SFD) and KC received an award from the Fondation de France.

\section{Disclosure of Potential Conflicts of Interest}

None of the authors has anything to disclose relevant to this article. 
Total word count: 5876

Number of figures: 2

Number of tables: 0

Abstract 160 mots

\begin{abstract}
Obesity is a chronic low-grade inflammatory disease (both at the systemic and adipose tissue level) that continues to rise worldwide. It is associated with an abundance of comorbidities, including type 2 diabetes (T2D). Bariatric surgery, which induces modifications of the intestinal tract, is to date the most successful treatment for obesity. Its use has dramatically increased in number as it enables both weight reduction and metabolic improvements, with $60 \%$ of patients even achieving diabetes remission. Several mechanisms are actually demonstrated to be involved in those clinical improvements. Importantly, both obesity and T2D share many phenotypic characteristics, including increased systemic and adipose tissue inflammation, as well as gut microbiota dysbiosis. These characteristics are deeply modulated after bariatric surgery. This review will specifically focus on the host metabolic changes induced by bariatric surgery in regards of the induced gut architectural changes, as well as on the modifications in the inflammatory tone and gut microbiota observed subsequently to bariatric surgery induced weight loss.
\end{abstract}

\title{
Keywords
}

Bariatric surgery, gut microbiota, inflammation, type 2 diabetes, obesity, microbiome 


\section{Introduction}

The obesity epidemic and its related-comorbidities, including metabolic alterations, Type 2 Diabetes (T2D), cardiovascular diseases and some cancers, continue to rise unabated ${ }^{1}$. Obesity is a low-grade inflammatory disease, in part due to inflammatory injuries in adipose tissue (AT) contributing eventually to moderate but chronic systemic inflammation (i.e. cytokines and circulating immune cells). Obesity-associated low-grade inflammation has a critical role in obesity-related complication development ${ }^{2}$. Major progresses have been made in understanding the timeline of events leading to the onset and maintenance of adipose tissue inflammation and its links with AT structural alterations and systemic metabolism, although this has been frequently explored on mouse models. Briefly, adipocyte size increases during weight gain and participates to inflammatory cytokine production (such as tumor necrosis factor alpha $(\mathrm{TNF} \alpha)$ ) and chemokines (as the monocyte chemoattractant protein 1 (MCP1) $)^{3,4}$, which recruit inflammatory cells within the AT. Macrophages ${ }^{5,6}$ probably account for the vast majority of AT inflammatory cells (as reviewed previously ${ }^{7}$ ) and display a mixed surface marker phenotype (M1 and M2) in human obese subjects ${ }^{8}$. Yet, they produce a myriad of pro- and anti-inflammatory cytokines ${ }^{9,10}$. Among the overall infiltrating AT macrophage, those organized in crown-like structures, which are also increased in obesity, are resolutely pro-inflammatory ${ }^{11}$. These cellular alterations in subcutaneous AT (scAT) are exacerbated in obese individuals with impaired glucose tolerance or $\mathrm{T} 2 \mathrm{D}^{6,11,12}$. Visceral AT (vAT), an adipose depot critically related to T2D and nonalcoholic fatty liver disease $(\mathrm{NAFLD})^{13}$, is more inflammatory than $\mathrm{scAT}^{5}$. Perturbations of adipose cellular cross-talk further contribute to maintain low-grade inflammation and altered metabolism. As such, adipocytes-macrophages cross-talks increases the inflammatory tone via 1) interleukin (IL) $1 \beta$ production by macrophages and inflammasome activation in the adipocytes ${ }^{14}$, and 2 ) 
macrophages toll-like receptor 4 (TLR-4) activation by adipocytes-released saturated freefatty acids ${ }^{4}$. IL1 $\beta$ production contributes to adipocyte insulin resistance.

In addition to macrophages, other inflammatory cell types, including lymphocytes ${ }^{15}$, mastocytes ${ }^{16}$ and neutrophils ${ }^{17}$, contribute to a perpetual inflammatory cycle and related perturbed adipose tissue metabolism in obesity. This includes, for example, an increased ratio of helper T cells 1:helper T cells 2 (Th1:Th2) that is associated with increased markers of insulin-resistance $^{18}$. Adipose tissue lymphocytes also produce IL-22 and IL-17, two interleukins that enhance macrophage IL-1 $\beta$ secretion in obese T2D patients ${ }^{15}$. Through IL$1 \beta$ secretion, macrophages, in return, induce the up-regulation of IL-22 and IL-17 surface receptors on lymphocytes ${ }^{4}$. The order of events has been suggested to be as follows: T-cells are recruited and activated within the AT, inducing macrophage accumulation, and this proinflammatory activation contribute to insulin-resistance development ${ }^{19}$. This sequence is nevertheless debated as reviewed $\mathrm{in}^{20}$.

Recently, the gut microbiota (GM) has emerged as a new contributor to weight gain and immune/inflammatory system imbalances. This concept has been demonstrated through fecal transfer experiments using mice ${ }^{21}$ or human ${ }^{22}$ donors and germ-free mice recipients, and suggests that fecal GM may impact energy metabolism and immune cell activation in the AT (reviewed in ${ }^{23}$ ). An increasing number of studies has further demonstrated that a nonnegligible proportion of obese subjects exhibit GM dysbiosis. This fecal GM phenotype is characterized by 1) decreased microbial gene richness $(M G R)^{24,25}$ that worsens with the severity of obesity and metabolic alterations ${ }^{26}$ and, 2) a switch in bacterial composition with a respective increase and decrease of species with pro-inflammatory and anti-inflammatory properties $^{25}$. Moreover, in genetically or high-fat diet induced obese mice, an increased intestinal permeability and subsequent translocation of bacteria and bacterial components into the systemic compartment was found to lead to increased circulating lipopolysaccharide 
(LPS) (termed “metabolic endotoxemia”) and accumulation of AT macrophages. Both dysbiosis and altered intestinal barrier may thus contribute to the inflammatory tone and related metabolic alterations ${ }^{27-29}$. In human obesity, however, this concept is still challenged as intestinal permeability is subtly altered in fasting conditions, but it appears to worsen dramatically after a lipid challenge and associates with increased systemic inflammation and $\mathrm{T} 2 \mathrm{D}^{30}$

Bariatric surgery (BS) represents a relevant model of rapid weight reduction combined with metabolic and inflammatory improvements. Recent data has also shown some improvements of GM dysbiosis, although only partial. However, whether bariatric surgeryinduced improved metabolism is firmly related to the changes of systemic and tissue inflammatory tone, as well as GM dysbiosis rescue, need detailed examination. In the current review, we focus on the effects of $\mathrm{BS}$ on T2D, GM and inflammation modifications and address whether these events can be connected.

\section{Bariatric surgery and Type 2 diabetes improvement}

Lifestyle modifications and/or dietary intervention are in general not sufficient to induce major and sustainable weight loss, and often result in weight regain ${ }^{31,32}$. The increasing prevalence of severe forms of obesity ${ }^{33}$ has been accompanied by a surge in BS interventions, with the number of surgeries increasing 2 -fold worldwide over the last 8 years ${ }^{34}$. Bariatric surgery is reserved for patients with a BMI $>40 \mathrm{~kg} / \mathrm{m}^{2}$ or a BMI $>35 \mathrm{~kg} / \mathrm{m}^{2}$ when associated with obesity related-diseases ${ }^{35}$. However, metabolic surgery has recently been proposed to T2D individual with BMI $>30 \mathrm{~kg} / \mathrm{m}^{2}$, yet this has not been implemented in every country's national guidelines ${ }^{36}$. Currently, BS can be divided into two main procedures: 1) either purely restrictive, with laparoscopic adjustable gastric banding (AGB) and vertical sleeve 
gastrectomy (VSG) or 2) using both restrictive and malabsorptive mechanisms, with Rouxen-Y gastric bypass (RYGB) and biliopancreatic diversion ${ }^{37}$. RYGB and VSG are the most widely performed types of BS worldwide ${ }^{34}$ and as such, we will focus on these procedures, whereas LAGB constitute a good weight loss control model. Although VSG and RYGB display low and similar rates of complications, mortality ${ }^{38}$ and comparable short term weight loss $^{39}$, RYGB tends to achieve greater weight loss and metabolic improvement in the longterm ${ }^{39}$.

An abundance of literature demonstrates the major beneficial effects of BS in inducing major and durable weight reduction ${ }^{40-42}$, in decreasing mortality ${ }^{43}$ and improving many obesity-related comorbidities. As T2D improves rapidly and more significantly following BS than with intensive medical therapy ${ }^{44}$, a new clinical concept has emerged: diabetes remission (DR), which is defined as the normalization of glycemic parameters in the absence of glucose lowering agents one year post-BS ${ }^{45}$. DR occurs in around $60 \%$ of T2D one year post-BS, yet this percentage varies according to type of surgery ${ }^{46}$. Randomized controlled trials have demonstrated that, compared to intensive medical care, RYGB and VSG both improve glucose control, as seen by the number of patients reaching their HbA1c target. However, post-RYGB, more patients are able to remain free of glucose lowering drugs (i.e. achieving DR) as compared to post-VSG patients ${ }^{47}$. Even in patients not reaching DR, a significant improvement of glycemic control is nevertheless observed, as evidenced by the reduction in $\mathrm{HbA}_{1} \mathrm{c}^{48,49}$ or the number of glucose lowering agents needed to reach the HbA1c target ${ }^{50}$. Despite these glucose metabolism improvements, however, T2D relapse occurs in the longerterm follow-up in $30 \%$ to $50 \%$ of the patients ${ }^{47,48,51,52}$ who had experienced DR at one year. Nevertheless, these patients still display improved glycemic control as compared to their metabolic status prior to BS. Poor weight loss at one year ${ }^{48}$, weight regain during the follow- 
up ${ }^{48,53}$, and T2D severity (represented by duration and number of glucose lowering agents) and duration prior to $\mathrm{BS}^{54,55}$ are among the different factors involved in T2D relapse.

Since DR following BS is not universal, there has been progress in developing scoring systems (DiaRem ${ }^{56}, \mathrm{ABCD}$ score $^{57}$, Ad-DiaRem ${ }^{58}$ and DiaBetter ${ }^{59}$ ) to predict patients' likelihood to achieve DR one year following surgery. These scores use several routine clinical parameters demonstrated to be involved with DR or non-DR. This includes age, T2D severity, and the quality of glycemic control assessed by HbA1c values. Studies have compared the predictive value of these scores ${ }^{58-60}$ and confirmed that whereas the DiaRem predicts well DR following surgery in certain individuals ${ }^{60}$, the Ad-DiaRem ${ }^{58}$ performed better for short term prediction (more individuals accurately predicted), but neither were sufficient to accurately predict longer-term outcomes ${ }^{54,61-63}$. A new score (5y-Ad-DiaRem) including weight loss during the first year post-BS has recently been shown to perform better than both the DiaRem and Ad-DiaRem to predict 5 years outcomes ${ }^{48}$. These clinical scores will be useful to select and propose different patient follow-ups or even future synergic therapies according to the different predicted outcomes.

\section{Proposed mechanisms of type 2 diabetes remission}

Several mechanisms have been suggested to participate in the beneficial metabolic outcomes post-BS. Le Roux et al., have summarized these effects as the "BRAVE effect", for Bile flow diversion, gastric size Reduction, Anatomical gut rearrangement, Vagal manipulation and Enteric gut hormone modulation ${ }^{64}$. Whereas those mechanisms are all observed post-RYGB, only some of them occur in VSG. The Figure 1 condenses the different mechanisms currently described as implicated in the metabolic improvements that are discussed below.

\section{Caloric restriction and weight loss}


Glucose levels improve within days post-RYGB ${ }^{65}$, suggesting that immediate postsurgery caloric restriction, independently of weight loss, is critical in T2D improvement. Energy intake is drastically reduced post-RYGB or VSG ${ }^{66-68}$, due to concomitant gastric pouch reduction ${ }^{69}$ and enhanced satiety following rapid changes in gut hormone secretion post-BS before any significant weight $\operatorname{loss}^{70,71}$. This includes the increase in anorexic hormone such as cholecystokinin $(\mathrm{CCK})^{72}$, peptide $\mathrm{YY}(\mathrm{PYY})^{73}$, glucagon-like peptide 1 $(\mathrm{GLP}-1)^{74}$, oxyntomodulin $(\mathrm{OXM})^{75}$ and eventually the decrease in the orexigenic hormone ghrelin $^{76}$.

Despite these findings in the absence of weight loss, the amount of weight loss per se also seems to explain post-BS improvements in glucose homeostasis. While a $10 \%$ weight loss induced either by $\mathrm{RYGB}^{74,77} \mathrm{LAGB}^{77}$ or a diet restriction ${ }^{74}$ improves glucose metabolism, RYGB improves $\beta$-cell function better than LAGB or dietary restriction, which is probably linked to the BRAVE effect. However, a more substantial weight loss (20\% of initial body weight) similarly improved $\beta$-cell function after both RYGB and LAGB ${ }^{77}$. The amount of weight loss also appears important in explaining DR after different surgical procedures as individuals with poor weight loss display reduced rates of $\mathrm{DR}^{51}$. However, mechanisms other than energy restriction or weight loss cannot be excluded when examining diabetes improvement post-BS ${ }^{65}$, and among them, early improvement of insulin sensitivity, specifically post-RYGB as compared to LAGB or a restrictive diet ${ }^{78}$, has been proposed.

\section{Changes in hormones and beta cell function}

In addition to their role in the control of food intake, the impact of gut hormones on insulin secretion and insulin resistance have been abundantly described in the literature ${ }^{79-81}$. The hindgut hypotheses (i.e. rapid emptying of the gastric pouch thus enabling the direct delivery of nutrients into the ileum) may explain the modulation of the gut endocrine system 
post-BS ${ }^{82-84}$. GLP-1, synthetized by the intestinal L-cells, remains the most explored of the gut hormones following BS. T2D patients display an altered incretin effect due to reduced post-prandial GLP-1 secretion ${ }^{85,86}$. The specific effects of GLP-1 on the pancreas include: 1 ) preventing $\beta$-cell death, 2) stimulating glucose-induced insulin secretion (GSIS), and 3) the expansion of $\beta$-cell mass ${ }^{87}$. Early following BS, post-prandial GLP-1 secretion is enhanced and may participate to improve insulin secretion ${ }^{74}$. However, the role of GLP-1 in explaining glucose metabolism improvements post-BS has been challenged. Indeed, blocking the GLP-1 receptor with the pharmacologist antagonist Exendin 9-39 amide in patients post-RYGB alters insulin secretion, but does not significantly modify glucose levels in either the fasting or fed state nor does it impact the subjects’ glucose tolerance during OGTT ${ }^{88,89}$. Similarly, the beneficial metabolic effects of BS are not altered in mice with genetic invalidation of the GLP-1 receptor ${ }^{90,91}$. Recent evidence proposes a more complex mechanism of GLP-1 mediating post-BP improvements in glucose metabolism. In mice with knock-out (KO) for the GLP-1 receptor specifically in $\beta$-cells, glucose metabolism is not rescued after VSG whereas it was normal in the WT counterpart. This observation suggests a contribution specifically localized GLP-1 receptors in explaining the metabolic effects of GLP-1 post$\mathrm{BS}^{92}$. An alternative hypothesis proposes that GLP-1 stimulation of GSIS depends mostly on the $\alpha$-cells paracrine GLP-1 secretion rather than that of intestinal L-cells. $\alpha$-cells can indeed secrete GLP-1 by switching the expression of their prohormone convertase (PC) (i.e. favoring PC 1/3 instead of PC 2) $)^{93,94}$, thus probably explaining, in part, the results obtained in specific $\beta$-cells GLP-1 receptor KO undergoing VSG ${ }^{92}$. Concordantly, VSG increases the $\alpha$-cells paracrine production of GLP-1, yet it still requires $\beta$-cells GLP-1 receptor presence ${ }^{95}$.

Literature on adaptive changes of pancreatic insulin secretion post-BS started with the exploration of subjects with hyperinsulinemic hypoglycemia complications post-RYGB. Service et al., described an increased $\beta$-cell mass (i.e. nesidioblastose) upon resecting the 
pancreas in 6 patients suffering from neuroglucopenic symptoms of post-prandial hypoglycemia post-RYGB ${ }^{96}$. Since then, whereas one study confirmed this first finding ${ }^{97}$, others observed no change in pancreatic $\beta$-cell mass ${ }^{98,99}$ post-BS. These diverging results might originate from the difficulty in directly assessing pancreatic tissue in humans. Some groups have thus evaluated $\beta$-cell mass or function using tomography or magnetic resonance imaging to assess pancreatic triglyceride content ${ }^{100}$ coupled with functional exploration (Cpeptide production and insulin sensibility) ${ }^{99}$. Rather than increase observed previously, a decrease in pancreatic $\beta$-cell mass was shown due to the reduction in pancreatic steatosis and decreased pancreatic insulin content, a phenomenon suggested to be linked to pancreas adaptation following weight loss. Similarly to heterogeneous findings in humans, animal model studies report either enhanced ${ }^{101,102}$ or decreased ${ }^{95,103,104} \beta$-cell mass post-BS. Differences in insulin-resistance status may explain such variability with decreased $\beta$-cell mass observed solely in humans or mice who predominantly improved their insulin sensibility. Nevertheless, despite the major improvement in glucose homeostasis, $\beta$-cell function is not rescued by BS, and remains dysfunctional as compared to lean normoglycemic subjects $^{105}$. Along with weight regain ${ }^{48}$, persistent $\beta$-cell dysfunction could participate in T2D relapse observed during follow-up, in agreement with findings demonstrating that T2D severity and duration ${ }^{48}$ contribute to diabetes non-remission.

Interestingly, Glucagon-like Peptide-2 (GLP-2) is also proposed to be involved in the metabolic improvements. GLP-2, co-expressed with GLP-1 in intestinal L-cells and released upon nutrient ingestion, has a beneficial trophic role on the small intestine with stimulation of crypt cell proliferation, increased bowel weight, villus growth of both the jejunum and the ileum ${ }^{106,107}$. Plasma GLP-2 significantly increases post-RYGB, concomitantly with increased crypt cell proliferation ${ }^{108,109}$; thus, GLP-2 may participate to the gut hypertrophy induced by BS. GLP-2 also displays a protective effect on gut barrier function and inflammation ${ }^{28}$. Using 
prebiotics in obese mice induces higher GLP-2 circulating concentration, lower plasma LPS and decreased systemic and hepatic inflammation together with metabolic improvements ${ }^{28}$. Pharmacologic agonist GLP-2 treatment reproduced the beneficial effect of the prebiotic treatment ${ }^{28}$. In humans, the therapeutic injection of GLP-2 in small bowel disease also results in significantly improved nutrient absorption, which is probably due to improvements in gut function and reduced inflammation ${ }^{110}$. Since GLP-1 and GLP-2 are co-secreted, one could wonder whether the physiological response to the anatomic changes induced by BS could be explained by enhanced GLP-2 secretion to restore the gut anatomy with GLP-1 secretion being merely driven by this adaptive effect.

\section{Enteroplasticity and gut remodeling}

Each section of the intestine is highly and functionally specialized, with nutrient absorption occurring generally in the proximal to mid-intestine (i.e. duodenum/ jejunum) whereas the gut-barrier and gut peptide secretion are likely more important along the distal intestine. The intestinal mucosa is organized into a stratified inner and a larger outer layer, which is where most commensal bacteria are hosted, thus enabling the cross-talk between the host and the environment. The intestine is highly plastic, as it has been shown that large surgical ileal removal ${ }^{111}$ induce an increased cellular proliferation to increase glucose uptake. Both RYGB and VSG modify the gut anatomy, and various mouse studies using different BS techniques have described an intestinal adaptation post-BS. Whereas the non-alimentary bypassed limb tends to atrophy ${ }^{112}$, both the alimentary and common limb show signs of hyperplasia (up to $40 \%$ weight gain, higher villus height and crypt depth, signs of proliferation, increased L-cell number without changes in L-cell density) post-RYGB ${ }^{108,112-}$ 114. By contrast, whereas VSG does not induce gut hypertrophy, it results in increased number and density of enteroendocrine L-cells ${ }^{115}$. 
The increased number of intestinal proglucagon-derived peptide secreting cells postBS agrees with the above described increase of gut hormones secretion post-BS. Despite hormonal changes, modifications of nutrient sensing by the remodeled gut are also suggested to be involved in improvement of glucose metabolism post-RYGB. Troy et al., suggested that duodenojejunal bypass enhanced intestinal neoglucogenesis and portal glucose sensing thus resulting in improved insulin sensitivity ${ }^{116}$. More recently, it was demonstrated that BSinduced gut remodeling is different according to the BS techniques. RYGB induces intestinal hyperplasia and increased glucose uptake through increased glucose transporter 1 (GLUT-1) at the basolateral membrane of enterocytes ${ }^{115}$ to support tissue growth ${ }^{117}$. By contrast, VSG is not associated with intestinal hyperplasia yet VSG mice display delayed glucose absorption. Finally, recent evidence demonstrates that bile diversion also plays a role in the decrease of intestinal glucose uptake post-RYGB ${ }^{118}$. Finally, since endogenous sodium contained in bile acids is necessary for glucose uptake by sodium glucose cotransporter 1 (SGLT1), it was recently demonstrated that glucose uptake (via SGLT1) occurred only in the common limb post-RYGB thus reducing the total intestine glucose uptake, and therefore improving plasma glucose.

\section{Bile acids}

Bile acids (BA) are synthetized in the liver, stored in the gallbladder, and delivered to the duodenum in response to nutrients. BA act as surfactants and play a critical role in lipid absorption. Furthermore, BA have a positive impact on pancreatic function since they are able to trigger GLP-1 secretion, through their action as natural ligands on the Takeda G protein coupled membrane receptor (TGR5) receptor expressed in intestinal L-cells ${ }^{119}$. Both RYGB and VSG induce modifications of fasting and post-prandial BA serum concentration and composition ${ }^{103,120-123}$ whereas LAGB solely reduces serum $\mathrm{BA}^{120}$. Although endoluminal concentration of BA post-BS does not seem to be modified in animal models ${ }^{124}$, this needs to 
be evaluated in humans, especially because the BA composition differs between rodents and human. Interestingly, intrajejunal bile infusion of taurocholic acid can experimentally lower blood glucose concentration and enhance GLP-1 secretion and C-peptide/glucose ratio ${ }^{125}$ in healthy men.

Indirect proof of the involvement of BA on weight loss and improved glucose tolerance has been demonstrated using TGR5 and farnesoid X receptor (FXR) KO mice submitted to VSG, BA being another natural ligand for $\mathrm{FXR}^{119}$. In $\mathrm{TGR}^{-/-}$mice, VSG fails to induce improvements in body weight, fasting glycaemia and glucose tolerance ${ }^{103}$; thus suggesting the importance of the TGR5 signaling pathway in the improvement of glucose homeostasis post-BS. The proposed mechanism of BA underlying BS-induced improvements in body weight and metabolism includes the reduction of systemic and hepatic inflammation, which would improve insulin signaling, but not an increase of GLP-1 or insulin secretion ${ }^{103}$. Furthermore, $\mathrm{FXR}^{-/-}$mice undergoing VSG display impaired reduction of fasting glycaemia and glucose tolerance ${ }^{122}$. This further suggests the importance of FXR in the improvement of glucose homeostasis post-BS. However, the complete physiological mechanisms are clouded by pharmacological studies administering agonists and antagonists of intestinal FXR displaying similar clinical effects. Indeed, both the inhibition of the intestinal FXR with either tauro-beta-muricholate (T-beta-MCA) ${ }^{126}$ or glycoursodeoxycholic acid (GUDCA) ${ }^{127}$, or the selective intestinal FXR agonist using fexaramine ${ }^{128}$ improve metabolic disorders in mice fed a HFD ${ }^{126-128}$. Notably, the improvement of glucose homeostasis in these pharmacological studies could merely originate from the induced weight loss or at least reduced weight gain and reduction of systemic inflammation ${ }^{128}$. While BA are ligands for FXR and TGR5, ultimately, the pool of bile acids (secondary vs. primary) are largely important for how BA affect these signaling pathways. Most importantly, BA can influence 
microbiota composition, and microbiota reciprocally influence the BA pool through $\mathrm{FXR}^{122,129}$, thus, it is important to examine BA in parallel with the gut microbiota.

\section{Change of gut microbiota after bariatric surgery}

\section{Gut microbiota compositional modifications}

Remarkable shifts in the gut microbiota (GM) composition have been consistently described post-BS in both short ${ }^{130-133}$ or long term ( $>5$ years) studies ${ }^{26,134}$, however there is a major inter-individual variability ${ }^{135}$. There is no doubt that those modifications might be partly driven by the drastic modulation of the gut architecture ${ }^{136}$ as well as food intake reduction ${ }^{24}$, hormonal and bile acid modification, and eventually, low-grade inflammation changes. Importantly, because drugs impact microbiota composition ${ }^{137}$, their change or even cessation due to BS-induced metabolic improvement might also account for major GM modifications post-BS. Yet, this has, to date, never been examined in BS studies. For example, metformin has largely been shown to influence GM composition in both mice ${ }^{138}$ and humans ${ }^{139}$. As some patients experience DR post-BS, they are able to discontinue their glucose-lowering agents following $\mathrm{BS}^{48}$, which could account for differences in GM profiles as compared to patients not achieving DR. Likewise, proton-pump inhibitors (PPIs), systematically prescribed during the first months post-BS, induce GM modifications ${ }^{140,141}$ by

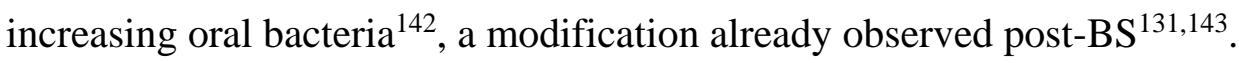

A recent meta-analysis ${ }^{132}$ summarized the changes in GM composition post-BS and showed that among the plethora of bacterial changes, two genera, Escherichia and Akkermansia, have been consistently reported to strongly increased post-BS ${ }^{132}$. Akkermansia muciniphila abundance is associated with insulin sensitivity and decreased inflammation in several studies ${ }^{144,145}$ exploring obese subjects. Metformin is also known to increase the abundance of both Akkermansia and Escherichia ${ }^{139,146,147}$, thus suggesting that BS could 
mimic some of the glucose-lowering effects metformin effects via the microbiome. Another bacteria, Faecalibacterium prausnitzii, increases solely in T2D obese individuals postRYGB, and this augmentation is correlated with the improvement of systemic inflammation ${ }^{130}$. However, whether the increase in the proportional abundance of these bacteria species in the GM is causally linked to improved glucose metabolism is unknown. Unpublished data from our team revealed that the marked increase in Akkermansia muciniphila seen after RYGB, but not LAGB, is not related to improved insulin-sensitivity markers (Dao et al., in revision), albeit in severely obese subjects with marked dysbiosis that only partially improves after BS. In this context, a hallmark of GM modifications post-BS is the increase in microbial gene richness $(\mathrm{MGR})^{26,131}$ with drastic amelioration of insulinsensitivity, adiposity, BMI and systemic inflammation ${ }^{24,26,131}$. However, morbidly obese patients post-BS do not normalize their bacterial richness in proportion to their metabolic improvements ${ }^{26}$.

In the context of T2D remission, only one study has focused on the comparison of microbial changes between patients experiencing or not experiencing DR post-BS ${ }^{148}$. Even though only 14 patients were examined (7 RYGB, 7 VSG), Murphy et al., showed that presurgical GM composition differed between patients who eventually experienced DR as compared to those who did not. Post-surgery, individuals experiencing DR had increasing levels of the genera Faecalibacterium (only RYGB), and the species Roseburia intestinalis (both RYGB and VSG), both of which have already been associated with decreased lowgrade inflammation and improved glucose homeostasis in both humans and rodents ${ }^{122,130}$.

Although there is no doubt that GM composition is significantly changing post-BS, this association does not 1) confirms the involvement of GM in metabolic improvements found post-BS nor 2) provides insights into the potentially mechanisms involved. To decipher 
the relationship between the host metabolic improvements, BS, and GM, fecal microbiota transplantation (FMT) appears a relevant tool to explore causality.

\section{Fecal transplantations effects}

FMT has been scarcely studied post-BS ${ }^{134,149,150}$, and only two studies provide information related to glucose homeostasis improvement ${ }^{149,150}$. FMT from either post-RYGB or sham-operated obese mice into germ-free mice leads to decreased adiposity in the animals receiving post-RYGB feces. A recent study performing FMT from human to mice confirmed this effect ${ }^{134}$. Germ-free mice receiving FMT from patients who had undergone BS up to 9 years before gained less adiposity than those receiving FMT from non-operated obese individuals ${ }^{134}$. Additionally, RYGB recipients displayed decreased circulating insulin levels and HOMA-IR, while the opposite could be observed for the Sham-recipient ${ }^{149}$, suggesting that post-BS GM could replicate some metabolic benefits of the surgery. Finally, Arora et al., performed two different FMT experiments from BS or sham operated fa/fa rats into germfree mice, transferring either the ileum or the caecum content as inoculum ${ }^{\mathbf{1 5 0}}$. They showed that the ileal FMT (from RYGB) increased both the $\alpha$-diversity and fat mass, yet altered their glucose tolerance as compared to that of ileal sham controls. By contrast, caecal FMT postRYGB induced a significant decrease in post-prandial glucose excursion, yet there was no significant effect on weight as compared to the caecal sham control FMT. This apparent discrepancy between these observations and those previously reported by Liou et al., could also originate from the differences in the models (mice vs. rats and diet-induced obesity vs. genetic obesity and T2D) ${ }^{\mathbf{1 4 9}}$. These promising yet conflicting results underline the need for replicative studies, and while further research is needed to fully determine the factors affecting GM composition following surgery, the switch in GM functionality may be a more important factor that impacts clinical outcomes post-BS. 


\section{Gut microbiota functional modification}

\section{Metabolic endotoxemia and gut barrier improvements}

As previously described, obesity and T2D is associated with increased gut permeability $^{27-29}$ in mice and humans. For the latter, this occurs mostly after a lipid challenge $^{30}$. This phenomenon is mainly driven by lipopolysaccharides (LPS), an outer membrane molecule of gram negative bacteria, which triggers inflammation via the TLR4/CD14 complex ${ }^{27-29}$. Literature has repeatedly reported the decrease of systemic LPS post-BS ${ }^{151-155}$, which has even been associated with improvements in insulin resistance and glucose control ${ }^{152,154}$. A recent murine study demonstrated that RYGB induced the improvement of tight-junction integrity and improved in-vivo intestinal permeability together with the reduction of metabolic endotoxemia and systemic inflammation ${ }^{156}$. This improved intestinal permeability could originate from 1 ) the observed reduction of fat intake post-BS ${ }^{157}$ as fat intake indeed alters the gut barrier ${ }^{27,30}$ or 2) the increased Akkermansia muciniphila observed post-BS. While this latter factor needs to be further explored, supplementing mice with alive or pasteurized $A$. muciniphila is able to reduce plasma LPS and is associated with improvements of the gut barrier as well as glucose metabolism ${ }^{138,158}$. Whether this is a possible mechanism in BS-induced metabolic improvement remains unknown.

\section{$\underline{\text { Bile acids }}$}

As mentioned above, BA have an impact on glucose homeostasis post-BS, and are strongly connected to GM composition. Experiments using germ-free or antibiotic-treated mice demonstrated the obligatory role of the GM to ensure the physiological production of unconjugated and secondary $\mathrm{BA}^{129,159}$. As $\mathrm{GM}$ dysbiosis contributes to altered BA metabolism in inflammatory bowel diseases (IBD), one could wonder whether the same is also occurring in other diseases presenting with GM dysbiosis, such as obesity ${ }^{145}$ or $\mathrm{T}^{2} \mathrm{D}^{160}$. 
Importantly, BS induces an increase in conjugated BA in humans and rodents ${ }^{103,120-123}$ which acts partly through FXR to improve glucose tolerance post-BS ${ }^{122}$. FXR KO mice submitted to VSG displayed differences in GM composition such as lower levels of Roseburia spp. ${ }^{122}$ as compared to their wild-type counterpart. Most importantly, GM functionality linked to BA metabolism was demonstrated to significantly differ between RYGB and obese individuals without surgery. Specifically, they observed an increased abundance of microbial genes involved in the production of secondary BA, concomitantly with increased post prandial levels of BA and fibroblast-growth factor 19 (FGF-19) post-RYGB, the latter of which is an intestinal hormone that regulates $\mathrm{BA}$ pools via $\mathrm{FXR}^{134}$. However, the link between the modifications of BA and GM had not been evaluated in this study. More recently, GUDCA has been demonstrated to be an intestinal FXR antagonist and is increased upon metformin treatment and improves glucose tolerance. Importantly, it was recently demonstrated that metformin improves glucose tolerance via the decrease of Bacteroides fragilis abundance which itself is correlated to GUDCA levels and acts through FXR signaling ${ }^{127}$. They further showed that FMT using feces of metformin-treated patients increases insulin-sensitivity and levels of intestinal tauro- $\beta$-muricholic acid (T $\beta \mathrm{MCA}$ ) in antibiotic-treated mice, whereas FMT using untreated patients was deleterious, similarly to the inoculation with $B$. fragilis which abrogated the beneficial effects of metformin on BA levels ${ }^{127}$. It is thus possible, yet unproven, that GM evolution post-BS is implicated in the observed increase of GUDCA or TßMCA, which further suggests a metformin-like action of BS on glucose homeostasis. Nevertheless, whether BS induces modification of Bacteroides fragilis and could therefore explain partly the improvement in glucose metabolism observed post-BS has still not been described to date. 


\section{$\underline{\text { Branched-chain amino acids }}$}

Branched-chain amino acids (BCAA) are essential amino-acids such as leucine, isoleucine and valine, consisting up to $20 \%$ of daily amino-acid intake. Although beneficial effects of BCAA supplementation have been described ${ }^{161-163}$, other studies also show increased BCAA levels in obesity ${ }^{164,165}$, insulin-resistance and $\mathrm{T}^{2} \mathrm{D}^{160,166-170}$. BCAA mediate IR through the activation of the mammalian target of rapamycin complex 1 (mTORC1) and its downstream target, the ribosomal protein S6 kinase beta-1 (S6K1). mTORC1 and S6K1 induce insulin-resistance via the inhibition of phosphorylation of the insulin-receptor (IRS-1) 171,172. BCAA are implicated in pancreatic beta-cell proliferation in the presence of increased glucose $^{173}$. Relationships between GM, serum BCAA and insulin-resistance are also suggested $^{165,166,174}$. Patients with insulin-resistance display increased levels of serum BCAA together with a GM enriched in Prevotella copri and Bacteroides vulgatus, which are two species harboring increased expression of genes involved in the biosynthesis of BCAAs and reduced expression of genes for internal BCAA use ${ }^{166}$. Most importantly, our team demonstrated that RYGB induces a reduction in serum BCAAs, demonstrating another potential mechanism of BS to improve glucose-related parameters ${ }^{26}$. This decrease in BCAAs is also observed post-BS in several other studies ${ }^{175-181}$. Moreover, RYGB also induces an improvement in microbial gene richness which was at baseline significantly associated with increased Bacteroides vulgatus ${ }^{26}$. Similarly, we found positive associations between this species and metabolic deterioration in severe obesity.

Most interestingly, Laferrère et al., demonstrated that BCAA decreased significantly solely in patients who lost at least 10 kilograms of body weight post-BS, but not in patients with a similar weight loss induced by a restrictive diet ${ }^{175}$, suggesting the role of a BSdependent mechanism in BCAA reduction. Whether the reduction in BCAA post-BS could be another weight-loss independent factor implicated in the resolution of T2D and 
implicating the GM would need demonstration. Indirect support of this is that reducing BCAAs have beneficial effects on glucose control independent of weight loss. As well, several experiments demonstrated that glucose-lowering drugs such as metformin ${ }^{182,183}$ and glipizide $^{183}$ promote the up-regulation of BCAA degradation pathways, also leading to a decreased serum BCAA ${ }^{182}$.

\section{Inflammatory changes post-BS: links with improvement in T2D?}

\section{Adipose tissue remodeling}

As depicted in Figure 2, BS enables a dramatic loss in body fat mass ${ }^{67}$ involving both from scAT and vAT depots ${ }^{5}$. It was initially described that AT undergoes significant remodeling during $\mathrm{BS}$ and that $\mathrm{BS}$ reduces adipocyte size, yet more effectively in DR patients ${ }^{184}$. Although the relationship has not been firmly confirmed, reduced adipocyte size might contribute to the reduced production of cytokines such as IL-1Ra ${ }^{185,186}$ or IL-8, IP-10, monokine-induced by $\gamma$-interferon (MIG), MCP-1, and "Regulated on Activation, Normal T cell expressed and secreted” (RANTES), along with the improvement of body weight and metabolic alterations. Moreover, BS is associated with fewer macrophages in the scAT ${ }^{186,187}$ and in the $\mathrm{vAT}^{188}$ and a switch toward a less pro-inflammatory profile ${ }^{8,187}$, which may also participate in the reduced inflammatory tone post-BS. T2D patients undergoing long-term DR lose more total fat mass and trunk-fat mass than those remaining T2D post-RYGB, suggesting a possible role of vAT modifications in $\mathrm{DR}^{48}$. This result is concordant with that of a previous study where the remission of metabolic syndrome (pre-diabetes state) was observed in patients who lost the most vAT mass post-RYGB ${ }^{189}$.

Notably, a recent study demonstrated that, while obese mice on a dietary restriction can normalize their body weight, they do not fully rescue their insulin sensitivity profile nor AT inflammation with the conservation of crown like structures. Moreover, macrophages still 
display an inflammatory profile with a perpetual cross talk with T-cells maintaining inflammatory activation ${ }^{190}$. These results, that need to be confirmed in human, suggest that although weight loss improves obesity-induced AT injuries, it does not restore its structure and function, which is important in the context of BS as some individuals do not normalize their BMI post-BS ${ }^{40,48}$. In addition, it is well known that BS induces major inter-individual variability in clinical outcomes and weight response, and that one could speculate that patients undergoing DR would improve AT inflammation and metabolism better than those not achieving DR and with persistent inflammatory alterations.

\section{Circulating immune and cytokine production changes}

Together with changes in inflammation in the AT, modifications of circulating immune cells and inflammatory cytokines are observed post-BS. Blood CD16+ monocytes, known to display an increased inflammatory cytokine production, are increased during obesity ${ }^{191}$ and positively correlates with $\mathrm{BMI}^{192}$ and the specific $\mathrm{CD} 14 \operatorname{dim} \mathrm{CD} 16+$ subpopulation further augments in obese individuals with T2D ${ }^{191}$. Interestingly, the CD14dim CD16+ subpopulation exhibits the lowest lipid accumulation capacity of all monocytes and is less prone to migrate into the $\mathrm{AT}^{193}$. BS significantly reduces these two subpopulations and, most particularly the CD14dim CD16+ monocytes ${ }^{191,194}$ while switching their secretory profile towards a decreased production of inflammatory cytokines such as TNF $\alpha$ and IL6 ${ }^{194}$. Interestingly, Chen et al., recently showed significant associations between changes in GM composition post-BS (assessed by RT-PCR) and the changes in monocytes number, secretion profile, and the expression of their membrane receptors such as TLR-4, which is the receptor that recognizes bacterially-produced LPS, thus involved in microbiota signalization pathway ${ }^{194}$.

Circulating T-lymphocytes display a Th1 inflammatory profile during obesity ${ }^{18}$ and also undergo phenotype modifications post-BS. Whereas the blood number of lymphocytes is not 
modified, Th1 cells and the Th1/Th2 ratio decreases significantly post-BS induced-weight loss $^{195}$ (Figure 2). This decrease is stronger among patients with T2D or impaired glucose tolerance (IGT) than normoglycemic patients pre-BS ${ }^{195}$. In agreement with a switch in inflammatory profile, the magnitude of glycemic improvement post-BS is correlated with the increase in Th2 post-BS in a clinical study focusing on T2D or IGT patients ${ }^{196}$. Blood levels of a specific T cell type, Mucosal-associated invariant T (MAIT) cells, are dramatically decreased in obese and obese T2D patients ${ }^{197,198}$ but display a Th1 and Th17 cytokine production profile ${ }^{197}$ while their number is increased in the AT. The relationship between MAIT cells and hyperglycemic milieu was substantiated by an in vitro study showing that hyperglycemia per se induces MAIT apoptosis ${ }^{198}$, which could partly explain their reduction during obesity and T2D. Interestingly, MAIT cells recognize microbial derived metabolites (originating from the riboflavin pathway) that are able to activate these cells. Recently, it was also demonstrated that MAIT cells could be activated by some bacterial species ${ }^{199}$. RYGB is able to 1) increase the number of MAIT cells, yet their level does not return to that of normal healthy controls and 2) nearly normalize their cytokine secretion profile with the exception of IL-17 ${ }^{197}$, concomitantly with weight loss and improvements in glycemic control. Overall, the inflammatory tone as well as the inflammatory profile of circulating and AT immune cells tend to decreased post-BS.

\section{Conclusions and perspectives}

In this review, we ought to describe the beneficial effects of BS in terms of glucose control improvement and diabetes remission, as well as the myriad of complex mechanisms probably acting in synergy to improve glucose homeostasis. BS induces caloric restriction, weight loss, fat-mass loss, improved $\beta$-cell function, gut plasticity, both hormonal and bile acids changes, as well as immune cell modifications at the systemic level and within the 
adipose tissue. However, the causal or secondary contribution of these factors in improved glucose metabolism still need to be deciphered. Recently, GM dysbiosis was shown to be partially improved after BS, however not to the same degree as metabolic improvements. Thus, whether the GM per se plays a role in DR observed post-BS still remains to be proven. Additionally, the mechanisms by which GM compositional and functional modifications post-BS participates in improved glucose metabolism and/or inflammatory tone still need to be evaluated in depth in controlled studies focusing on the combination of mechanisms proposed above in this review. Exploring these interactions will also help identifying targets for biomarkers or upstream therapeutics mimicking BS effects without surgical intervention. 


\section{Figures legends}

Figure 1. Mechanisms involved in type-2 diabetes remission after bariatric surgery.

GLP-1: Glucagon-like peptide 1; GLP-2: Glucagon-like peptine 2; PYY: Peptide YY; OXM: Oxyntomodulin; CCK: Cholecystokinin; GLUT1: Glucose transporter 1; GUDCA: Glycoursodeoxycholic acid; T $\beta$ MCA: Tauro- $\beta$-muricholic acid; SGLT1: Sodium-glucose linked transporter 1; LPS: Lipopolysaccharide; $\mathrm{Na}^{+}$: Sodium; RYGB: Roux-en-Y gastric bypass; VSG: Vertical sleeve gastrectomy.

Figure 2. Immunity and inflammation profiles of adipose tissue before and after bariatric surgery.

VAT: visceral adipose tissue; scAT: subcutaneous adipose tissue; Th1: helper T cells 1; Th2: helper T cells 2 . 


\section{References}

1. Kopelman, P. G. Obesity as a medical problem. Nature 404, 635-643 (2000).

2. Tordjman, J. et al. Association between omental adipose tissue macrophages and liver histopathology in morbid obesity: influence of glycemic status. J. Hepatol. 51, 354-362 (2009).

3. Skurk, T., Alberti-Huber, C., Herder, C. \& Hauner, H. Relationship between adipocyte size and adipokine expression and secretion. J. Clin. Endocrinol. Metab. 92, 1023-1033 (2007).

4. Engin, A. B. Adipocyte-Macrophage Cross-Talk in Obesity. Adv. Exp. Med. Biol. 960, 327-343 (2017).

5. Cancello, R. et al. Increased infiltration of macrophages in omental adipose tissue is associated with marked hepatic lesions in morbid human obesity. Diabetes 55, 15541561 (2006).

6. Harman-Boehm, I. et al. Macrophage infiltration into omental versus subcutaneous fat across different populations: effect of regional adiposity and the comorbidities of obesity. J. Clin. Endocrinol. Metab. 92, 2240-2247 (2007).

7. Dalmas, E., Clément, K. \& Guerre-Millo, M. Defining macrophage phenotype and function in adipose tissue. Trends Immunol. 32, 307-314 (2011).

8. Aron-Wisnewsky, J. et al. Human adipose tissue macrophages: $\mathrm{m} 1$ and $\mathrm{m} 2$ cell surface markers in subcutaneous and omental depots and after weight loss. J. Clin. Endocrinol. Metab. 94, 4619-4623 (2009).

9. $\quad$ Lumeng, C. N., Bodzin, J. L. \& Saltiel, A. R. Obesity induces a phenotypic switch in adipose tissue macrophage polarization. J. Clin. Invest. 117, 175-184 (2007). 
10. Huber, J. et al. CC chemokine and CC chemokine receptor profiles in visceral and subcutaneous adipose tissue are altered in human obesity. J. Clin. Endocrinol. Metab. 93, 3215-3221 (2008).

11. Wentworth, J. M. et al. Pro-inflammatory CD11c+CD206+ adipose tissue macrophages are associated with insulin resistance in human obesity. Diabetes 59, 1648-1656 (2010).

12. Pasarica, M. et al. Lower total adipocyte number but no evidence for small adipocyte depletion in patients with type 2 diabetes. Diabetes Care 32, 900-902 (2009).

13. Després, J.-P. \& Lemieux, I. Abdominal obesity and metabolic syndrome. Nature 444, 881-887 (2006).

14. Stienstra, R. et al. Inflammasome is a central player in the induction of obesity and insulin resistance. Proc. Natl. Acad. Sci. U. S. A. 108, 15324-15329 (2011).

15. Dalmas, E. et al. T cell-derived IL-22 amplifies IL-1 $\beta$-driven inflammation in human adipose tissue: relevance to obesity and type 2 diabetes. Diabetes 63, 1966-1977 (2014).

16. Divoux, A. et al. Mast cells in human adipose tissue: link with morbid obesity, inflammatory status, and diabetes. J. Clin. Endocrinol. Metab. 97, E1677-1685 (2012).

17. Rouault, C. et al. Roles of chemokine ligand-2 (CXCL2) and neutrophils in influencing endothelial cell function and inflammation of human adipose tissue. Endocrinology 154, 1069-1079 (2013).

18. Viardot, A. et al. Obesity is associated with activated and insulin resistant immune cells. Diabetes Metab. Res. Rev. 28, 447-454 (2012).

19. Nishimura, S. et al. CD8+ effector T cells contribute to macrophage recruitment and adipose tissue inflammation in obesity. Nat. Med. 15, 914-920 (2009).

20. Sell, H., Habich, C. \& Eckel, J. Adaptive immunity in obesity and insulin resistance. Nat. Rev. Endocrinol. 8, 709-716 (2012). 
21. Bäckhed, F. et al. The gut microbiota as an environmental factor that regulates fat storage. Proc. Natl. Acad. Sci. U. S. A. 101, 15718-15723 (2004).

22. Ridaura, V. K. et al. Gut microbiota from twins discordant for obesity modulate metabolism in mice. Science 341, 1241214 (2013).

23. Bohan, R. et al. Gut microbiota: a potential manipulator for host adipose tissue and energy metabolism. J. Nutr. Biochem. 64, 206-217 (2018).

24. Cotillard, A. et al. Dietary intervention impact on gut microbial gene richness. Nature 500, 585-588 (2013).

25. Le Chatelier, E. et al. Richness of human gut microbiome correlates with metabolic markers. Nature 500, 541-546 (2013).

26. Aron-Wisnewsky, J. et al. Major microbiota dysbiosis in severe obesity: fate after bariatric surgery. Gut (2018). doi:10.1136/gutjnl-2018-316103

27. Cani, P. D. et al. Metabolic endotoxemia initiates obesity and insulin resistance. Diabetes 56, 1761-1772 (2007).

28. Cani, P. D. et al. Changes in gut microbiota control inflammation in obese mice through a mechanism involving GLP-2-driven improvement of gut permeability. Gut 58, 10911103 (2009).

29. Cani, P. D. et al. Changes in gut microbiota control metabolic endotoxemia-induced inflammation in high-fat diet-induced obesity and diabetes in mice. Diabetes 57, 1470_ $1481(2008)$.

30. Genser, L. et al. Increased jejunal permeability in human obesity is revealed by a lipid challenge and is linked to inflammation and type 2 diabetes. J. Pathol. (2018). doi:10.1002/path.5134

31. Dyson, P. A. The therapeutics of lifestyle management on obesity. Diabetes Obes. Metab. 12, 941-946 (2010). 
32. Wing, R. R. \& Phelan, S. Long-term weight loss maintenance. Am. J. Clin. Nutr. 82, 222S-225S (2005).

33. Sturm, R. \& Hattori, A. Morbid obesity rates continue to rise rapidly in the United States. Int. J. Obes. 2005 37, 889-891 (2013).

34. Angrisani, L. et al. Bariatric Surgery and Endoluminal Procedures: IFSO Worldwide Survey 2014. Obes. Surg. 27, 2279-2289 (2017).

35. Fried, M. et al. Interdisciplinary European guidelines on metabolic and bariatric surgery. Obes. Surg. 24, 42-55 (2014).

36. Rubino, F. et al. Metabolic Surgery in the Treatment Algorithm for Type 2 Diabetes: A Joint Statement by International Diabetes Organizations. Diabetes Care 39, 861-877 (2016).

37. Dixon, J. B., Straznicky, N. E., Lambert, E. A., Schlaich, M. P. \& Lambert, G. W. Surgical approaches to the treatment of obesity. Nat. Rev. Gastroenterol. Hepatol. 8, 429-437 (2011).

38. Zellmer, J. D., Mathiason, M. A., Kallies, K. J. \& Kothari, S. N. Is laparoscopic sleeve gastrectomy a lower risk bariatric procedure compared with laparoscopic Roux-en-Y gastric bypass? A meta-analysis. Am. J. Surg. 208, 903-910; discussion 909-910 (2014).

39. Osland, E., Yunus, R. M., Khan, S., Memon, B. \& Memon, M. A. Weight Loss Outcomes in Laparoscopic Vertical Sleeve Gastrectomy (LVSG) Versus Laparoscopic Roux-en-Y Gastric Bypass (LRYGB) Procedures: A Meta-Analysis and Systematic Review of Randomized Controlled Trials. Surg. Laparosc. Endosc. Percutan. Tech. 27, 8-18 (2017).

40. Sjöström, L. et al. Bariatric surgery and long-term cardiovascular events. JAMA J. Am. Med. Assoc. 307, 56-65 (2012). 
41. Adams, T. D. et al. Weight and Metabolic Outcomes 12 Years after Gastric Bypass. N. Engl. J. Med. 377, 1143-1155 (2017).

42. Courcoulas, A. P. et al. Seven-Year Weight Trajectories and Health Outcomes in the Longitudinal Assessment of Bariatric Surgery (LABS) Study. JAMA Surg. (2017). doi:10.1001/jamasurg.2017.5025

43. Adams, T. D. et al. Long-term mortality after gastric bypass surgery. N. Engl. J. Med. 357, 753-761 (2007).

44. Schauer, P. R., Mingrone, G., Ikramuddin, S. \& Wolfe, B. Clinical Outcomes of Metabolic Surgery: Efficacy of Glycemic Control, Weight Loss, and Remission of Diabetes. Diabetes Care 39, 902-911 (2016).

45. Buse, J. B. et al. How do we define cure of diabetes? Diabetes Care 32, 2133-2135 (2009).

46. Pournaras, D. J. et al. Effect of the definition of type II diabetes remission in the evaluation of bariatric surgery for metabolic disorders. Br. J. Surg. 99, 100-103 (2012).

47. Schauer, P. R. et al. Bariatric Surgery versus Intensive Medical Therapy for Diabetes 5-Year Outcomes. N. Engl. J. Med. 376, 641-651 (2017).

48. Debédat, J. et al. Long-term Relapse of Type 2 Diabetes After Roux-en-Y Gastric Bypass: Prediction and Clinical Relevance. Diabetes Care (2018). doi:10.2337/dc180567

49. Mingrone, G. et al. Bariatric surgery versus conventional medical therapy for type 2 diabetes. N. Engl. J. Med. 366, 1577-1585 (2012).

50. Thereaux, J. et al. Long-term follow-up after bariatric surgery in a national cohort. Br. J. Surg. 104, 1362-1371 (2017). 
51. Mingrone, G. et al. Bariatric-metabolic surgery versus conventional medical treatment in obese patients with type 2 diabetes: 5 year follow-up of an open-label, single-centre, randomised controlled trial. The Lancet 386, 964-973 (2015).

52. Courcoulas, A. P. et al. Three-Year Outcomes of Bariatric Surgery vs Lifestyle Intervention for Type 2 Diabetes Mellitus Treatment: A Randomized Clinical Trial. JAMA Surg. 150, 931-940 (2015).

53. Brethauer, S. A. et al. Can diabetes be surgically cured? Long-term metabolic effects of bariatric surgery in obese patients with type 2 diabetes mellitus. Ann. Surg. 258, 628636; discussion 636-637 (2013).

54. Aminian, A., Brethauer, S. A., Kashyap, S. R., Kirwan, J. P. \& Schauer, P. R. DiaRem score: external validation. Lancet Diabetes Endocrinol. 2, 12-13 (2014).

55. Mehaffey, J. H. et al. Type 2 diabetes remission following gastric bypass: does diarem stand the test of time? Surg. Endosc. 31, 538-542 (2017).

56. Still, C. D. et al. Preoperative prediction of type 2 diabetes remission after Roux-en-Y gastric bypass surgery: a retrospective cohort study. Lancet Diabetes Endocrinol. 2, 3845 (2014).

57. Lee, W.-J. et al. Predicting success of metabolic surgery: age, body mass index, Cpeptide, and duration score. Surg. Obes. Relat. Dis. Off. J. Am. Soc. Bariatr. Surg. 9, 379-384 (2013).

58. Aron-Wisnewsky, J. et al. The advanced-DiaRem score improves prediction of diabetes remission 1 year post-Roux-en-Y gastric bypass. Diabetologia (2017). doi:10.1007/s00125-017-4371-7

59. Pucci, A. et al. Type 2 diabetes remission 2 years post Roux-en-Y gastric bypass and sleeve gastrectomy: the role of the weight loss and comparison of DiaRem and DiaBetter scores. Diabet. Med. J. Br. Diabet. Assoc. 35, 360-367 (2018). 
60. Cotillard, A. et al. Type 2 Diabetes Remission After Gastric Bypass: What Is the Best Prediction Tool for Clinicians? Obes. Surg. 25, 1128-1132 (2015).

61. Lee, M. H. et al. Predictors of long-term diabetes remission after metabolic surgery. $J$. Gastrointest. Surg. Off. J. Soc. Surg. Aliment. Tract 19, 1015-1021 (2015).

62. Wood, G. C., Mirshahi, T., Still, C. D. \& Hirsch, A. G. Association of DiaRem Score With Cure of Type 2 Diabetes Following Bariatric Surgery. JAMA Surg. 151, 779-781 (2016).

63. Dicker, D., Yahalom, R., Comaneshter, D. S. \& Vinker, S. Long-Term Outcomes of Three Types of Bariatric Surgery on Obesity and Type 2 Diabetes Control and Remission. Obes. Surg. 26, 1814-1820 (2016).

64. Ashrafian, H. et al. Diabetes resolution and hyperinsulinaemia after metabolic Roux-enY gastric bypass. Obes. Rev. Off. J. Int. Assoc. Study Obes. 12, e257-272 (2011).

65. Pories, W. J. et al. Who would have thought it? An operation proves to be the most effective therapy for adult-onset diabetes mellitus. Ann. Surg. 222, 339-350; discussion 350-352 (1995).

66. Laurenius, A. et al. Changes in eating behaviour and meal pattern following Roux-en-Y gastric bypass. Int. J. Obes. 2005 36, 348-355 (2012).

67. Verger, E. O. et al. Micronutrient and Protein Deficiencies After Gastric Bypass and Sleeve Gastrectomy: a 1-year Follow-up. Obes. Surg. (2015). doi:10.1007/s11695-015$1803-7$

68. Aron-Wisnewsky, J. et al. Nutritional and Protein Deficiencies in the Short Term following Both Gastric Bypass and Gastric Banding. PloS One 11, e0149588 (2016).

69. Gras-Miralles, B. et al. Caloric intake capacity as measured by a standard nutrient drink test helps to predict weight loss after bariatric surgery. Obes. Surg. 24, 2138-2144 (2014). 
70. le Roux, C. W. et al. Gut hormone profiles following bariatric surgery favor an anorectic state, facilitate weight loss, and improve metabolic parameters. Ann. Surg. 243, 108-114 (2006).

71. Lips, M. A. et al. Calorie restriction is a major determinant of the short-term metabolic effects of gastric bypass surgery in obese type 2 diabetic patients. Clin. Endocrinol. (Oxf.) 80, 834-842 (2014).

72. Steinert, R. E. et al. Ghrelin, CCK, GLP-1, and PYY(3-36): Secretory Controls and Physiological Roles in Eating and Glycemia in Health, Obesity, and After RYGB. Physiol. Rev. 97, 411-463 (2017).

73. Yousseif, A. et al. Differential effects of laparoscopic sleeve gastrectomy and laparoscopic gastric bypass on appetite, circulating acyl-ghrelin, peptide YY3-36 and active GLP-1 levels in non-diabetic humans. Obes. Surg. 24, 241-252 (2014).

74. Laferrère, B. et al. Effect of weight loss by gastric bypass surgery versus hypocaloric diet on glucose and incretin levels in patients with type 2 diabetes. J. Clin. Endocrinol. Metab. 93, 2479-2485 (2008).

75. Laferrère, B. et al. Rise of oxyntomodulin in response to oral glucose after gastric bypass surgery in patients with type 2 diabetes. J. Clin. Endocrinol. Metab. 95, 40724076 (2010).

76. Cummings, D. E. et al. Plasma ghrelin levels after diet-induced weight loss or gastric bypass surgery. N. Engl. J. Med. 346, 1623-1630 (2002).

77. Holter, M. M. et al. Glucose Metabolism After Gastric Banding and Gastric Bypass in Individuals With Type 2 Diabetes: Weight Loss Effect. Diabetes Care 40, 7-15 (2017).

78. Gastaldelli, A. et al. Short-Term Effects of Laparoscopic Adjustable Gastric Banding Versus Roux-en-Y Gastric Bypass. Diabetes Care dc152823 (2016). doi:10.2337/dc152823 
79. Dimitriadis, G. K., Randeva, M. S. \& Miras, A. D. Potential Hormone Mechanisms of Bariatric Surgery. Curr. Obes. Rep. 6, 253-265 (2017).

80. Laferrère, B. \& Pattou, F. Weight-Independent Mechanisms of Glucose Control After Roux-en-Y Gastric Bypass. Front. Endocrinol. 9, (2018).

81. Castagneto Gissey, L., Casella Mariolo, J. \& Mingrone, G. Intestinal peptide changes after bariatric and minimally invasive surgery: Relation to diabetes remission. Peptides 100, 114-122 (2018).

82. Wang, G. et al. Accelerated gastric emptying but no carbohydrate malabsorption 1 year after gastric bypass surgery (GBP). Obes. Surg. 22, 1263-1267 (2012).

83. Stano, S. et al. Effect of meal size and texture on gastric pouch emptying and glucagonlike peptide 1 after gastric bypass surgery. Surg. Obes. Relat. Dis. Off. J. Am. Soc. Bariatr. Surg. 13, 1975-1983 (2017).

84. Jacobsen, S. H. et al. Effects of gastric bypass surgery on glucose absorption and metabolism during a mixed meal in glucose-tolerant individuals. Diabetologia 56, 2250-2254 (2013).

85. Vilsbøll, T., Krarup, T., Deacon, C. F., Madsbad, S. \& Holst, J. J. Reduced postprandial concentrations of intact biologically active glucagon-like peptide 1 in type 2 diabetic patients. Diabetes 50, 609-613 (2001).

86. Knop, F. K. et al. Reduced incretin effect in type 2 diabetes: cause or consequence of the diabetic state? Diabetes 56, 1951-1959 (2007).

87. Xu, G., Stoffers, D. A., Habener, J. F. \& Bonner-Weir, S. Exendin-4 stimulates both beta-cell replication and neogenesis, resulting in increased beta-cell mass and improved glucose tolerance in diabetic rats. Diabetes 48, 2270-2276 (1999). 
88. Vetter, M. L. et al. GLP-1 plays a limited role in improved glycemia shortly after Rouxen-Y gastric bypass: a comparison with intensive lifestyle modification. Diabetes 64, 434-446 (2015).

89. Shah, M. et al. Contribution of endogenous glucagon-like peptide 1 to glucose metabolism after Roux-en-Y gastric bypass. Diabetes 63, 483-493 (2014).

90. Mokadem, M., Zechner, J. F., Margolskee, R. F., Drucker, D. J. \& Aguirre, V. Effects of Roux-en-Y gastric bypass on energy and glucose homeostasis are preserved in two mouse models of functional glucagon-like peptide-1 deficiency. Mol. Metab. 3, 191-201 (2014).

91. Wilson-Pérez, H. E. et al. Vertical sleeve gastrectomy is effective in two genetic mouse models of glucagon-like Peptide 1 receptor deficiency. Diabetes 62, 2380-2385 (2013).

92. Garibay, D. et al. $\beta$-Cell Glucagon-Like Peptide-1 Receptor Contributes to Improved Glucose Tolerance After Vertical Sleeve Gastrectomy. Endocrinology 157, 3405-3409 (2016).

93. Chambers, A. P. et al. The Role of Pancreatic Preproglucagon in Glucose Homeostasis in Mice. Cell Metab. 25, 927-934.e3 (2017).

94. Marchetti, P. et al. A local glucagon-like peptide 1 (GLP-1) system in human pancreatic islets. Diabetologia 55, 3262-3272 (2012).

95. Garibay, D. et al. $\beta$ Cell GLP-1R Signaling Alters $\alpha$ Cell Proglucagon Processing after Vertical Sleeve Gastrectomy in Mice. Cell Rep. 23, 967-973 (2018).

96. Service, G. J. et al. Hyperinsulinemic hypoglycemia with nesidioblastosis after gastricbypass surgery. N. Engl. J. Med. 353, 249-254 (2005).

97. Dadheech, N., Garrel, D. \& Buteau, J. Evidence of unrestrained beta-cell proliferation and neogenesis in a patient with hyperinsulinemic hypoglycemia after gastric bypass surgery. Islets 10, 213-220 (2018). 
98. Meier, J. J., Butler, A. E., Galasso, R. \& Butler, P. C. Hyperinsulinemic Hypoglycemia After Gastric Bypass Surgery Is Not Accompanied by Islet Hyperplasia or Increased Cell Turnover. Diabetes Care 29, 1554-1559 (2006).

99. Lautenbach, A. et al. Adaptive changes in pancreas post Roux-en-Y gastric bypass induced weight loss. Diabetes Metab. Res. Rev. 34, e3025 (2018).

100. Gaborit, B. et al. Ectopic fat storage in the pancreas using 1H-MRS: importance of diabetic status and modulation with bariatric surgery-induced weight loss. Int. J. Obes. 2005 39, 480-487 (2015).

101. Zhou, X. et al. Pancreatic hyperplasia after gastric bypass surgery in a GK rat model of non-obese type 2 diabetes. J. Endocrinol. 228, 13-23 (2016).

102. Zhang, S. et al. Increased $\beta$-Cell Mass in Obese Rats after Gastric Bypass: A Potential Mechanism for Improving Glycemic Control. Med. Sci. Monit. 23, 2151-2158 (2017).

103. McGavigan, A. K. et al. TGR5 contributes to glucoregulatory improvements after vertical sleeve gastrectomy in mice. Gut 66, 226-234 (2017).

104. Cummings, B. P. et al. Bile-acid-mediated decrease in endoplasmic reticulum stress: a potential contributor to the metabolic benefits of ileal interposition surgery in UCDT2DM rats. Dis. Model. Mech. 6, 443-456 (2013).

105. Dutia, R. et al. Limited recovery of $\beta$-cell function after gastric bypass despite clinical diabetes remission. Diabetes 63, 1214-1223 (2014).

106. Drucker, D. J. et al. Regulation of the biological activity of glucagon-like peptide 2 in vivo by dipeptidyl peptidase IV. Nat. Biotechnol. 15, 673-677 (1997).

107. Drucker, D. J., Erlich, P., Asa, S. L. \& Brubaker, P. L. Induction of intestinal epithelial proliferation by glucagon-like peptide 2. Proc. Natl. Acad. Sci. U. S. A. 93, 7911-7916 (1996). 
108. le Roux, C. W. et al. Gut hypertrophy after gastric bypass is associated with increased glucagon-like peptide 2 and intestinal crypt cell proliferation. Ann. Surg. 252, 50-56 (2010).

109. Cazzo, E. et al. GLP-1 and GLP-2 Levels are Correlated with Satiety Regulation After Roux-en-Y Gastric Bypass: Results of an Exploratory Prospective Study. Obes. Surg. 27, 703-708 (2017).

110. Jeppesen, P. B. et al. Glucagon-like peptide 2 improves nutrient absorption and nutritional status in short-bowel patients with no colon. Gastroenterology 120, 806-815 (2001).

111. Iqbal, C. W., Qandeel, H. G., Zheng, Y., Duenes, J. A. \& Sarr, M. G. Mechanisms of ileal adaptation for glucose absorption after proximal-based small bowel resection. $J$. Gastrointest. Surg. Off. J. Soc. Surg. Aliment. Tract 12, 1854-1864; discussion 18641865 (2008).

112. Li, B., Lu, Y., Srikant, C. B., Gao, Z.-H. \& Liu, J.-L. Intestinal adaptation and Reg gene expression induced by antidiabetic duodenal-jejunal bypass surgery in Zucker fatty rats. Am. J. Physiol. Gastrointest. Liver Physiol. 304, G635-645 (2013).

113. Taqi, E. et al. The influence of nutrients, biliary-pancreatic secretions, and systemic trophic hormones on intestinal adaptation in a Roux-en-Y bypass model. J. Pediatr. Surg. 45, 987-995 (2010).

114. Cavin, J.-B. et al. Malabsorption and intestinal adaptation after one anastomosis gastric bypass compared with Roux-en-Y gastric bypass in rats. Am. J. Physiol.-Gastrointest. Liver Physiol. 311, G492-G500 (2016).

115. Cavin, J.-B. et al. Differences in Alimentary Glucose Absorption and Intestinal Disposal of Blood Glucose After Roux-en-Y Gastric Bypass vs Sleeve Gastrectomy. Gastroenterology 150, 454-464.e9 (2016). 
116. Troy, S. et al. Intestinal gluconeogenesis is a key factor for early metabolic changes after gastric bypass but not after gastric lap-band in mice. Cell Metab. 8, 201-211 (2008).

117. Saeidi, N. et al. Reprogramming of intestinal glucose metabolism and glycemic control in rats after gastric bypass. Science 341, 406-410 (2013).

118. Baud, G. et al. Bile Diversion in Roux-en-Y Gastric Bypass Modulates SodiumDependent Glucose Intestinal Uptake. Cell Metab. 23, 547-553 (2016).

119. Thomas, C. et al. TGR5-mediated bile acid sensing controls glucose homeostasis. Cell Metab. 10, 167-177 (2009).

120. Kohli, R. et al. Weight loss induced by Roux-en-Y gastric bypass but not laparoscopic adjustable gastric banding increases circulating bile acids. J. Clin. Endocrinol. Metab. 98, E708-712 (2013).

121. Patti, M.-E. et al. Serum bile acids are higher in humans with prior gastric bypass: potential contribution to improved glucose and lipid metabolism. Obes. Silver Spring Md 17, 1671-1677 (2009).

122. Ryan, K. K. et al. FXR is a molecular target for the effects of vertical sleeve gastrectomy. Nature 509, 183-188 (2014).

123. Albaugh, V. L. et al. Early Increases in Bile Acids Post Roux-en-Y Gastric Bypass Are Driven by Insulin-Sensitizing, Secondary Bile Acids. J. Clin. Endocrinol. Metab. 100, E1225-E1233 (2015).

124. Duboc, H. et al. Roux-en-Y Gastric-Bypass and sleeve gastrectomy induces specific shifts of the gut microbiota without altering the metabolism of bile acids in the intestinal lumen. Int. J. Obes. 1 (2018). doi:10.1038/s41366-018-0015-3 
125. Wu, T. et al. Effects of Taurocholic Acid on Glycemic, Glucagon-like Peptide-1, and Insulin Responses to Small Intestinal Glucose Infusion in Healthy Humans. J. Clin. Endocrinol. Metab. 98, E718-E722 (2013).

126. Li, F. et al. Microbiome remodelling leads to inhibition of intestinal farnesoid $\mathrm{X}$ receptor signalling and decreased obesity. Nat. Commun. 4, 2384 (2013).

127. Sun, L. et al. Gut microbiota and intestinal FXR mediate the clinical benefits of metformin. Nat. Med. 1 (2018). doi:10.1038/s41591-018-0222-4

128. Fang, S. et al. Intestinal FXR agonism promotes adipose tissue browning and reduces obesity and insulin resistance. Nat. Med. 21, 159-165 (2015).

129. Sayin, S. I. et al. Gut Microbiota Regulates Bile Acid Metabolism by Reducing the Levels of Tauro-beta-muricholic Acid, a Naturally Occurring FXR Antagonist. Cell Metab. 17, 225-235 (2013).

130. Furet, J.-P. et al. Differential Adaptation of Human Gut Microbiota to Bariatric Surgery-Induced Weight Loss. Diabetes 59, 3049-3057 (2010).

131. Kong, L.-C. et al. Gut microbiota after gastric bypass in human obesity: increased richness and associations of bacterial genera with adipose tissue genes. Am. J. Clin. Nutr. 98, 16-24 (2013).

132. Guo, Y. et al. Modulation of the gut microbiome: a systematic review of the effect of bariatric surgery. Eur. J. Endocrinol. EJE-17-0403 (2017). doi:10.1530/EJE-17-0403

133. Graessler, J. et al. Metagenomic sequencing of the human gut microbiome before and after bariatric surgery in obese patients with type 2 diabetes: correlation with inflammatory and metabolic parameters. Pharmacogenomics J. 13, 514-522 (2013).

134. Tremaroli, V. et al. Roux-en-Y Gastric Bypass and Vertical Banded Gastroplasty Induce Long-Term Changes on the Human Gut Microbiome Contributing to Fat Mass Regulation. Cell Metab. 22, 228-238 (2015). 
135. Zhang, H. et al. Human gut microbiota in obesity and after gastric bypass. Proc. Natl. Acad. Sci. U. S. A. 106, 2365-2370 (2009).

136. Aron-Wisnewsky, J., Doré, J. \& Clement, K. The importance of the gut microbiota after bariatric surgery. Nat. Rev. Gastroenterol. Hepatol. 9, 590-598 (2012).

137. Rothschild, D. et al. Environment dominates over host genetics in shaping human gut microbiota. Nature 555, 210-215 (2018).

138. Shin, N.-R. et al. An increase in the Akkermansia spp. population induced by metformin treatment improves glucose homeostasis in diet-induced obese mice. Gut 63, 727-735 (2014).

139. Forslund, K. et al. Disentangling the effects of type 2 diabetes and metformin on the human gut microbiota. Nature 528, 262-266 (2015).

140. Jackson, M. A. et al. Gut microbiota associations with common diseases and prescription medications in a population-based cohort. Nat. Commun. 9, 2655 (2018).

141. Vesper, B. et al. The Effect of Proton Pump Inhibitors on the Human Microbiota. Curr. Drug Metab. 10, 84-89 (2009).

142. Imhann, F. et al. Proton pump inhibitors affect the gut microbiome. Gut 65, 740-748 (2016).

143. Ward, E. K. et al. The effect of PPI use on human gut microbiota and weight loss in patients undergoing laparoscopic Roux-en-Y gastric bypass. Obes. Surg. 24, 1567-1571 (2014).

144. Plovier, H. et al. A purified membrane protein from Akkermansia muciniphila or the pasteurized bacterium improves metabolism in obese and diabetic mice. Nat. Med. (2016). doi:10.1038/nm.4236 
145. Dao, M. C. et al. Akkermansia muciniphila and improved metabolic health during a dietary intervention in obesity: relationship with gut microbiome richness and ecology. Gut gutjnl-2014-308778 (2015). doi:10.1136/gutjnl-2014-308778

146. Cuesta-Zuluaga, J. de la et al. Metformin Is Associated With Higher Relative Abundance of Mucin-Degrading Akkermansia muciniphila and Several Short-Chain Fatty Acid-Producing Microbiota in the Gut. Diabetes Care 40, 54-62 (2017).

147. Shin, N.-R. et al. An increase in the Akkermansia spp. population induced by metformin treatment improves glucose homeostasis in diet-induced obese mice. Gut 63, 727-735 (2014).

148. Murphy, R. et al. Laparoscopic Sleeve Gastrectomy Versus Banded Roux-en-Y Gastric Bypass for Diabetes and Obesity: a Prospective Randomised Double-Blind Trial. Obes. Surg. 1-10 (2017). doi:10.1007/s11695-017-2872-6

149. Liou, A. P. et al. Conserved Shifts in the Gut Microbiota Due to Gastric Bypass Reduce Host Weight and Adiposity. Sci. Transl. Med. 5, 178ra41 (2013).

150. Arora, T. et al. Diabetes-associated microbiota in fa/fa rats is modified by Roux-en-Y gastric bypass. ISME J. 11, 2035-2046 (2017).

151. Casselbrant, A., Elias, E., Fändriks, L. \& Wallenius, V. Expression of tight-junction proteins in human proximal small intestinal mucosa before and after Roux-en-Y gastric bypass surgery. Surg. Obes. Relat. Dis. 11, 45-53 (2015).

152. Monte, S. V. et al. Reduction in endotoxemia, oxidative and inflammatory stress, and insulin resistance after Roux-en-Y gastric bypass surgery in patients with morbid obesity and type 2 diabetes mellitus. Surgery 151, 587-593 (2012).

153. Yang, P.-J. et al. Bariatric surgery decreased the serum level of an endotoxin-associated marker: lipopolysaccharide-binding protein. Surg. Obes. Relat. Dis. 10, 1182-1187 (2014). 
154. Trøseid, M. et al. Plasma Lipopolysaccharide Is Closely Associated With Glycemic Control and Abdominal Obesity: Evidence from bariatric surgery. Diabetes Care 36, 3627-3632 (2013).

155. Clemente-Postigo, M. et al. Lipopolysaccharide and lipopolysaccharide-binding protein levels and their relationship to early metabolic improvement after bariatric surgery. Surg. Obes. Relat. Dis. Off. J. Am. Soc. Bariatr. Surg. (2014). doi:10.1016/j.soard.2014.11.030

156. Guo, Y., Liu, C.-Q., Liu, G.-P., Huang, Z.-P. \& Zou, D.-J. Roux-en-Y gastric bypass decreases endotoxemia and inflammatory stress in association with improvement of gut permeability in obese diabetic rats. J. Diabetes (2019). doi:10.1111/1753-0407.12906

157. le Roux, C. W. et al. Gastric bypass reduces fat intake and preference. Am. J. Physiol.Regul. Integr. Comp. Physiol. 301, R1057-R1066 (2011).

158. Plovier, H. et al. A purified membrane protein from Akkermansia muciniphila or the pasteurized bacterium improves metabolism in obese and diabetic mice. Nat. Med. 23, 107-113 (2017).

159. Kuribayashi, H., Miyata, M., Yamakawa, H., Yoshinari, K. \& Yamazoe, Y. Enterobacteria-mediated deconjugation of taurocholic acid enhances ileal farnesoid $\mathrm{X}$ receptor signaling. Eur. J. Pharmacol. 697, 132-138 (2012).

160. Qin, J. et al. A metagenome-wide association study of gut microbiota in type 2 diabetes. Nature 490, 55-60 (2012).

161. Torres-Leal, F. L. et al. Leucine supplementation improves adiponectin and total cholesterol concentrations despite the lack of changes in adiposity or glucose homeostasis in rats previously exposed to a high-fat diet. Nutr. Metab. 8, 62 (2011).

162. Cota, D. et al. Hypothalamic mTOR signaling regulates food intake. Science 312, 927930 (2006). 
163. Binder, E. et al. Leucine supplementation modulates fuel substrates utilization and glucose metabolism in previously obese mice. Obes. Silver Spring Md 22, 713-720 (2014).

164. Newgard, C. B. et al. A branched-chain amino acid-related metabolic signature that differentiates obese and lean humans and contributes to insulin resistance. Cell Metab. 9, 311-326 (2009).

165. Ridaura, V. K. et al. Gut Microbiota from Twins Discordant for Obesity Modulate Metabolism in Mice. Science 341, 1241214 (2013).

166. Pedersen, H. K. et al. Human gut microbes impact host serum metabolome and insulin sensitivity. Nature 535, 376-381 (2016).

167. Stancáková, A. et al. Hyperglycemia and a common variant of GCKR are associated with the levels of eight amino acids in 9,369 Finnish men. Diabetes 61, 1895-1902 (2012).

168. Cheng, S. et al. Metabolite profiling identifies pathways associated with metabolic risk in humans. Circulation 125, 2222-2231 (2012).

169. Lanza, I. R. et al. Quantitative Metabolomics by 1H-NMR and LC-MS/MS Confirms Altered Metabolic Pathways in Diabetes. PLOS ONE 5, e10538 (2010).

170. Wang, T. J. et al. Metabolite profiles and the risk of developing diabetes. Nat. Med. 17, 448-453 (2011).

171. Yoon, M.-S. The Emerging Role of Branched-Chain Amino Acids in Insulin Resistance and Metabolism. Nutrients 8, (2016).

172. Melnik, B. C. Leucine signaling in the pathogenesis of type 2 diabetes and obesity. World J. Diabetes 3, 38-53 (2012).

173. Morales, N. B. \& Plata, C. A. de. Role of AKT/mTORC1 pathway in pancreatic $\beta$-cell proliferation. Colomb. Médica 43, 235-243-243 (2012). 
174. Wei, X. et al. Abnormal fecal microbiota community and functions in patients with hepatitis B liver cirrhosis as revealed by a metagenomic approach. BMC Gastroenterol. 13, 175 (2013).

175. Laferrère, B. et al. Differential Metabolic Impact of Gastric Bypass Surgery Versus Dietary Intervention in Obese Diabetic Subjects Despite Identical Weight Loss. Sci. Transl. Med. 3, 80re2-80re2 (2011).

176. Gralka, E. et al. Metabolomic fingerprint of severe obesity is dynamically affected by bariatric surgery in a procedure-dependent manner. Am. J. Clin. Nutr. 102, 1313-1322 (2015).

177. Lips, M. A. et al. Roux-en-Y Gastric Bypass Surgery, but Not Calorie Restriction, Reduces Plasma Branched-Chain Amino Acids in Obese Women Independent of Weight Loss or the Presence of Type 2 Diabetes. Diabetes Care 37, 3150-3156 (2014).

178. Lopes, T. I. B. et al. “Omics” Prospective Monitoring of Bariatric Surgery: Roux-En-Y Gastric Bypass Outcomes Using Mixed-Meal Tolerance Test and Time-Resolved 1H NMR-Based Metabolomics. OMICS J. Integr. Biol. 20, 415-423 (2016).

179. Magkos, F. et al. Effect of Roux-en-Y Gastric Bypass and Laparoscopic Adjustable Gastric Banding on Branched-Chain Amino Acid Metabolism. Diabetes 62, 2757-2761 (2013).

180. Narath, S. H. et al. An Untargeted Metabolomics Approach to Characterize Short-Term and Long-Term Metabolic Changes after Bariatric Surgery. PloS One 11, e0161425 (2016).

181. Arora, T. et al. Roux-en-Y Gastric Bypass Surgery Induces Early Plasma Metabolomic and Lipidomic Alterations in Humans Associated with Diabetes Remission. PLOS ONE 10, e0126401 (2015). 
182. De Haes, W. et al. Metformin promotes lifespan through mitohormesis via the peroxiredoxin PRDX-2. Proc. Natl. Acad. Sci. 111, E2501-E2509 (2014).

183. Walford, G. A. et al. Branched chain and aromatic amino acids change acutely following two medical therapies for type 2 diabetes mellitus. Metabolism 62, 17721778 (2013).

184. Cotillard, A. et al. Adipocyte size threshold matters: link with risk of type 2 diabetes and improved insulin resistance after gastric bypass. J. Clin. Endocrinol. Metab. 99, E1466-1470 (2014).

185. Dalmas, E. et al. Variations in circulating inflammatory factors are related to changes in calorie and carbohydrate intakes early in the course of surgery-induced weight reduction. Am. J. Clin. Nutr. 94, 450-458 (2011).

186. Cancello, R. et al. Reduction of macrophage infiltration and chemoattractant gene expression changes in white adipose tissue of morbidly obese subjects after surgeryinduced weight loss. Diabetes 54, 2277-2286 (2005).

187. Liu, Y. et al. Accumulation and Changes in Composition of Collagens in Subcutaneous Adipose Tissue After Bariatric Surgery. J. Clin. Endocrinol. Metab. 101, 293-304 (2016).

188. García-Rubio, J. et al. Cytometric analysis of adipose tissue reveals increments of adipocyte progenitor cells after weight loss induced by bariatric surgery. Sci. Rep. 8, 15203 (2018).

189. Faria, G. et al. Metabolic score: insights on the development and prediction of remission of metabolic syndrome after gastric bypass. Ann. Surg. 260, 279-286 (2014).

190. Zamarron, B. F. et al. Macrophage Proliferation Sustains Adipose Tissue Inflammation in Formerly Obese Mice. Diabetes db160500 (2016). doi:10.2337/db16-0500 
191. Poitou, C. et al. CD14dimCD16+ and CD14+CD16+ monocytes in obesity and during weight loss: relationships with fat mass and subclinical atherosclerosis. Arterioscler. Thromb. Vasc. Biol. 31, 2322-2330 (2011).

192. Rogacev, K. S. et al. Monocyte heterogeneity in obesity and subclinical atherosclerosis. Eur. Heart J. 31, 369-376 (2010).

193. Pecht, T. et al. Circulating Blood Monocyte Subclasses and Lipid-Laden Adipose Tissue Macrophages in Human Obesity. PloS One 11, e0159350 (2016).

194. Chen, H. et al. Change in gut microbiota is correlated with alterations in the surface molecule expression of monocytes after Roux-en-Y gastric bypass surgery in obese type 2 diabetic patients. 12

195. Viardot, A., Lord, R. V. \& Samaras, K. The Effects of Weight Loss and Gastric Banding on the Innate and Adaptive Immune System in Type 2 Diabetes and Prediabetes. J. Clin. Endocrinol. Metab. 95, 2845-2850 (2010).

196. Samaras, K., Viardot, A., Botelho, N. K., Jenkins, A. \& Lord, R. V. Immune cellmediated inflammation and the early improvements in glucose metabolism after gastric banding surgery. Diabetologia 56, 2564-2572 (2013).

197. Magalhaes, I. et al. Mucosal-associated invariant T cell alterations in obese and type 2 diabetic patients. J. Clin. Invest. 125, 1752-1762 (2015).

198. Touch, S. et al. Mucosal-associated invariant T (MAIT) cells are depleted and prone to apoptosis in cardiometabolic disorders. FASEB J. Off. Publ. Fed. Am. Soc. Exp. Biol. fj201800052RR (2018). doi:10.1096/fj.201800052RR

199. Tastan, C. et al. Tuning of human MAIT cell activation by commensal bacteria species and MR1-dependent T-cell presentation. Mucosal Immunol. 11, 1591-1605 (2018). 
Weight and fat-mass loss 3

\section{Endocrine pancreatic function 2}

- $\alpha$ cells: GLP-1 paracrine secretion

- $B$ cells: changed 6 cell mass?

\section{Alimentary limb remodeling}

$\uparrow$ Gut hormone secretion (1) (2)

GLP-1 (secreted along GLP-2), PYY, OXM and CCK

$\uparrow$ Nutrient sensing ( $\uparrow$ intestinal neoglucogenesis and

$\uparrow$ enterocyte glucose uptake (via GLUT1))

\section{Bile acids (BA)}

$\uparrow$ circulating and luminal conjugated BA (GUDCA, TBMCA) 3 *

$\uparrow$ Salt segregation in the biliopancreatic limb

$\downarrow$ Total glucose reabsorption via SGLT1 receptors

\section{Gut microbiota}

个 Gene richness 23

$\uparrow$ Abundances of certain species (Roseburia spp., Akkermansia spp., Escherichia spp., Faecalibacterium spp.) $23(4$

$\downarrow$ Circulating LPS 3

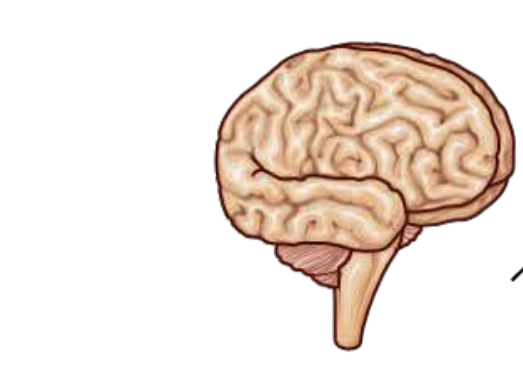

个 satiety

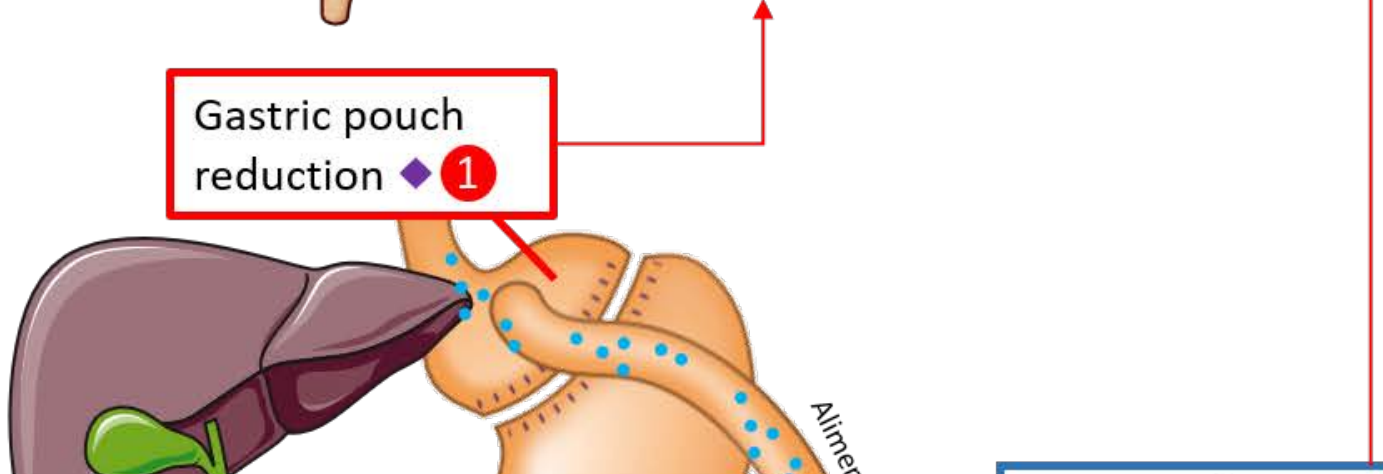

$\uparrow$ Villi height and crypt depth

$\uparrow$ Signs of proliferation

$\uparrow$ L-cell number

$\downarrow$ Gut permeability

$\downarrow$ branched chain amino-acids 


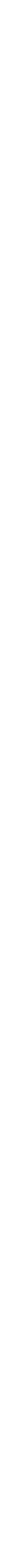

